ANALYSIS \& PDE Volume $5 \quad$ No. $2 \quad 2012$

ASYMPTOTIC DECAY FOR A ONE-DIMENSIONAL NONLINEAR
WAVE EQUATION

ASYMPTOTIC DECAY FOR A ONE-DIMENSIONAL NONLINEAR
WAVE EQUATION

\author{
HANS LindBLAD AND TERENCE TAO
}




\title{
ASYMPTOTIC DECAY FOR A ONE-DIMENSIONAL NONLINEAR WAVE EQUATION
}

\author{
HANS LINDBLAD AND TERENCE TAO
}

\begin{abstract}
We consider the asymptotic behaviour of finite energy solutions to the one-dimensional defocusing nonlinear wave equation $-u_{t t}+u_{x x}=|u|^{p-1} u$, where $p>1$. Standard energy methods guarantee global existence, but do not directly say much about the behaviour of $u(t)$ as $t \rightarrow \infty$. Note that in contrast to higher-dimensional settings, solutions to the linear equation $-u_{t t}+u_{x x}=0$ do not exhibit decay, thus apparently ruling out perturbative methods for understanding such solutions. Nevertheless, we will show that solutions for the nonlinear equation behave differently from the linear equation, and more specifically that we have the average $L^{\infty}$ decay $\lim _{T \rightarrow+\infty} \frac{1}{T} \int_{0}^{T}\|u(t)\|_{L_{x}^{\infty}(\mathbb{R})} d t=0$, in sharp contrast to the linear case. An unusual ingredient in our arguments is the classical Rademacher differentiation theorem that asserts that Lipschitz functions are almost everywhere differentiable.
\end{abstract}

\section{Introduction}

Fix $p>1$. We consider solutions $u: \mathbb{R} \times \mathbb{R} \rightarrow \mathbb{R}$ to the one-dimensional defocusing nonlinear wave equation

$$
-u_{t t}+u_{x x}=|u|^{p-1} u
$$

with the finite energy initial condition

$$
\|u(0)\|_{H_{x}^{1}(\mathbb{R})}+\left\|u_{t}(0)\right\|_{L_{x}^{2}(\mathbb{R})}<\infty .
$$

Standard energy methods (using the Sobolev embedding $H_{x}^{1} \subset L_{x}^{\infty}$ ) show that the initial value problem is locally well-posed in this energy class. Furthermore, by using the conservation of energy ${ }^{1}$

$$
E[u]=E[u(t)]:=\int_{\mathbb{R}} \mathbb{T}_{00}(t, x) d x,
$$

where $\mathbb{T}_{00}$ is the energy density

$$
\mathbb{T}_{00}:=\frac{1}{2} u_{t}^{2}+\frac{1}{2} u_{x}^{2}+\frac{1}{p+1}|u|^{p+1},
$$

Lindblad is supported by NSF grant DMS-0801120. Tao is supported by NSF Research Award DMS-0649473, the NSF Waterman award and a grant from the MacArthur Foundation.

MSC2010: 35L05.

Keywords: nonlinear wave equation.

${ }^{1}$ In order to justify energy conservation for solutions which are in the energy class, one can use standard local well-posedness theory to approximate such solutions by classical (i.e., smooth and compactly supported) solutions (regularising the nonlinearity $|u|^{p-1} u$ if necessary), derive energy conservation for the classical solutions, and then take strong limits. We omit the standard details. More generally, we shall perform manipulations such as integration by parts on finite energy solutions as if they were classical without any further comment. 
it is easy to show that the $H_{x}^{1} \times L_{x}^{2}$ norm of $u(t)$ does not blow up in finite time, and that the solution to (1) can be continued globally in time.

In this paper we study the asymptotic behaviour of finite energy solutions $u$ to (1) as $t \rightarrow \pm \infty$. Of course, from the conservation of energy (2) we know that $u(t)$ stays bounded in $\dot{H}_{x}^{1}(\mathbb{R}) \cap L_{x}^{p+1}(\mathbb{R})$, and thus (by the Gagliardo-Nirenberg inequality) bounded in $L_{x}^{\infty}(\mathbb{R})$ for all time, but this does not settle the question of whether $\|u(t)\|_{L_{x}^{\infty}(\mathbb{R})}$ exhibits any decay as $t \rightarrow \pm \infty$.

For the linear equation $-u_{t t}+u_{x x}=0$, the solutions are of course travelling waves $u(t, x)=f(x+t)+$ $g(x-t)$, which do not decay along light rays $x=x_{0} \pm t$. In particular, for any nontrivial linear solution, $\|u(t)\|_{L_{x}^{\infty}(\mathbb{R})}$ stays bounded away from zero. It is thus natural to ask whether the same behaviour occurs for solutions to the nonlinear Equation (1). However, an easy energy argument shows that the behaviour must be slightly different. Indeed, if we introduce the momentum density (or energy current)

$$
\mathbb{T}_{01}=\mathbb{T}_{10}:=u_{t} u_{x}
$$

and the momentum current

$$
\mathbb{T}_{11}:=\frac{1}{2} u_{t}^{2}+\frac{1}{2} u_{x}^{2}-\frac{1}{p+1},|u|^{p+1}
$$

we observe the conservation laws

$$
\begin{aligned}
& \partial_{t} \mathbb{T}_{00}=\partial_{x} \mathbb{\mathbb { T }}_{01}, \\
& \partial_{t} \mathbb{T}_{01}=\partial_{x} \mathbb{\mathbb { T }}_{11} \text {. }
\end{aligned}
$$

From (3) and the fundamental theorem of calculus we have

$$
\partial_{t} \int_{x<x_{0}+t} \mathbb{T}_{00}(t, x) d x=\mathbb{T}_{00}\left(t, x_{0}+t\right)+\mathbb{T}_{01}\left(t, x_{0}+t\right)
$$

for all $x_{0}, t \in \mathbb{R}$. On the other hand, from the nonnegativity of $\mathbb{T}_{00}$ we clearly have

$$
0 \leq \int_{x<x_{0}+t} \mathbb{T}_{00}(t, x) d x \leq E[u]
$$

From the fundamental theorem of calculus (and the monotone convergence theorem), we thus obtain

$$
\int_{-\infty}^{\infty} \mathbb{T}_{00}\left(t, x_{0}+t\right)+\mathbb{T}_{01}\left(t, x_{0}+t\right) d t \leq E[u]
$$

for all $x_{0} \in \mathbb{R}$. From the pointwise inequality $\mathbb{T}_{00}+\mathbb{T}_{01} \geq \frac{1}{p+1}|u|^{p+1}$ we conclude in particular the nonlinear decay estimate

$$
\int_{-\infty}^{\infty}|u|^{p+1}\left(t, x_{0}+t\right) d t \leq(p+1) E[u]
$$

for any $x_{0} \in \mathbb{R}$. From reflection symmetry we also have

$$
\int_{-\infty}^{\infty}|u|^{p+1}\left(t, x_{0}-t\right) d t \leq(p+1) E[u]
$$

for any $x_{0} \in \mathbb{R}$. We thus see that solutions to the nonlinear equation $u$ must decay (on average, at least) along any light ray $x=x_{0} \pm t$, in sharp contrast to solutions to the linear equation. This simple calculation 
already reveals that the nonlinear equation has somewhat different asymptotic behaviour from the linear equation, and in particular that it is highly unlikely that one can asymptotically analyse the former as a perturbation of the latter. This is in contrast with the one-dimensional nonlinear Klein-Gordon equation, for which the decay can be leveraged to obtain asymptotic results; see for instance [Lindblad and Soffer 2005]. Another contrast is with the local theory, which asserts that singularities for the nonlinear wave equation propagate along the same light rays as for the linear one; see [Reed 1978].

The estimates (5), (6) imply that finite energy solutions $u$ cannot concentrate on light rays $\left\{\left(t, x_{0} \pm t\right)\right.$ : $t \in \mathbb{R}\}$. However, it is a priori conceivable that such solutions might still concentrate on other worldlines $\{(t, x(t)): t \in \mathbb{R}\}$. Concentration on spacelike worldlines (in which $\left|x^{\prime}(t)\right|>1$ ) are easily ruled out by finite speed of propagation (or by a modification of the arguments used to derive (5), (6)), but concentration on timelike worldlines (in which $\left|x^{\prime}(t)\right|<1$ ) are not so obviously ruled out. Nevertheless, we are able to rule out this scenario by the following theorem, which is the main result of this paper.

Theorem 1.1 (Average $L_{x}^{\infty}$ decay). Let $u$ be a finite energy solution to (1), with an upper bound $E[u] \leq E$ on the energy. Then

$$
\frac{1}{2 T} \int_{t_{0}-T}^{t_{0}+T}\|u(t)\|_{L_{x}^{\infty}(\mathbb{R})} d t \leq c_{E, p}(T)
$$

for all $t_{0} \in \mathbb{R}$ and $T>0$, where $c_{E, p}: \mathbb{R}^{+} \rightarrow \mathbb{R}^{+}$is a function depending only on the energy bound $E$ and the exponent $p$ such that $c_{E, p}(t) \rightarrow 0$ as $t \rightarrow \infty$. In particular, we have

$$
\lim _{T \rightarrow+\infty} \sup _{t_{0} \in \mathbb{R}} \frac{1}{2 T} \int_{t_{0}-T}^{t_{0}+T}\|u(t)\|_{L_{x}^{\infty}(\mathbb{R})} d t=0 .
$$

The proof of this theorem will use energy estimates combined with a version of the Rademacher differentiation theorem (or Lebesgue differentiation theorem), that Lipschitz functions are almost everywhere differentiable. The basic idea is to observe that if $u$ concentrates on a timelike worldline $\{(t, x(t)): t \in \mathbb{R}\}$, then $x$ should be Lipschitz, and thus mostly differentiable. This implies that $u$ concentrates on certain parallelograms in spacetime; we will then use energy estimates to rule out such concentration.

In principle, the decaying bound $c_{E, p}(T)$ could be made explicit, but this would require a quantitative version of the Rademacher differentiation theorem. Such results exist (see [Tao 2009] or [Tao 2008, Section 2.4]), but they are fairly weak (involving the inverse tower exponential function $\log _{*}$ ). Presumably a more refined argument than the one given in this paper would give better bounds. For instance, it is plausible to conjecture that $\|u(t)\|_{L_{x}^{\infty}(\mathbb{R})}$ should decay at a polynomial rate in $t$, at least in the perturbative regime when $u$ is small.

We remark that our methods do not seem to give any precise asymptotics for the solution. Of course Theorem 1.1 indicates that the solution will not scatter to a linear solution, but it is not clear what the solution scatters to instead, even in the perturbative regime. It may be that techniques from nonlinear geometric optics could be useful to settle this question, but the extremely weak decay of the solution means that it would be very difficult for these methods to be made rigorous, at least until one can improve the results of Theorem 1.1 significantly. 


\section{Energy estimates}

In this section we derive the basic energy estimates needed to establish Theorem 1.1. Henceforth we fix $p$ and the finite energy solution $u$. We adopt the notation $X \lesssim Y$ or $X=O(Y)$ to denote the estimate $|X| \leq C Y$, where $C$ can depend on $p$ and the energy bound $E$. Thus from energy conservation we obtain the bounds

$$
\int_{\mathbb{R}}\left|u_{t}\right|^{2}(t, x)+\left|u_{x}\right|^{2}(t, x)+|u|^{p+1}(t, x) d x \lesssim 1
$$

for all $t$.

Lemma 2.1 (Hölder continuity). For all $t, x, t^{\prime}, x^{\prime} \in \mathbb{R}$ we have the pointwise bound

$$
u(t, x)=O(1)
$$

and the Hölder continuity property

$$
u(t, x)-u\left(t^{\prime}, x^{\prime}\right)=O\left(\left|t-t^{\prime}\right|^{1 / 2}+\left|x-x^{\prime}\right|^{1 / 2}\right) .
$$

Proof. The bound (8) follows immediately from (7) and the Gagliardo-Nirenberg inequality. Using the bound on $\left|u_{x}\right|^{2}$ in (7) together with the fundamental theorem of calculus and the Cauchy-Schwarz inequality, we also have the spatial Hölder continuity bound

$$
u(t, x)-u\left(t, x^{\prime}\right)=O\left(\left|x-x^{\prime}\right|^{1 / 2}\right) .
$$

Thus to prove (9) it will suffice to show that

$$
u\left(t_{1}, x_{0}\right)-u\left(t_{2}, x_{0}\right)=O\left(\left(t_{2}-t_{1}\right)^{1 / 2}\right)
$$

for all $t_{2}>t_{1}$. In view of (8) we may also assume $t_{2}=t_{1}+O(1)$.

Fix $t_{1}, t_{2}$. From (4) and the fundamental theorem of calculus we have

$$
\partial_{t} \int_{x<x_{0}} \mathbb{T}_{01}(t, x) d x=\mathbb{T}_{11}\left(t, x_{0}\right) ;
$$

integrating this in time and using (7) we obtain the bounds

$$
\int_{t_{1}}^{t_{2}} \mathbb{T}_{11}\left(t, x_{0}\right) d t=O(1) .
$$

Combining this with (8) we conclude

$$
\int_{t_{1}}^{t_{2}} u_{t}\left(t, x_{0}\right)^{2} d t=O(1)
$$

and (10) follows from the fundamental theorem of calculus and Cauchy-Schwarz.

Now we prove a more advanced energy estimate. 
Proposition 2.2 (nonlinear energy decay in a parallelogram). Let $T \geq R \geq 1$, let $x_{0}, t_{0} \in \mathbb{R}$, and let $v \in \mathbb{R}$ be a velocity. Then we have

$$
\int_{t_{0}-T}^{t_{0}+T} \int_{x_{0}+v t-R}^{x_{0}+v t+R}|u(t, x)|^{p+1} d x d t \lesssim R^{1 / 2} T^{1 / 2}+\frac{T}{R} .
$$

Remark 2.3. Energy conservation (7) only gives the bound of $O(T)$ for this integral, thus this proposition is nontrivial when $T$ is much larger than $R$. A key point here is that the bounds do not blow up in the neighbourhood of the speed of light $v=1$. It may be possible to improve the right-hand side of (11), and to also control other components of the energy, but the above bound will suffice for our purposes.

Proof. By translation invariance we can set $x_{0}=t_{0}=0$. By reflection symmetry we may assume that $v \geq 0$.

Let $\chi: \mathbb{R} \rightarrow \mathbb{R}$ be a nonnegative bump function supported on $[-2,2]$ which equals 1 on $[-1,1]$, and let $\psi(x):=\int_{y<x} \chi(y) d y$ be the antiderivative of $\chi$. From (4) and integration by parts we have

$$
\partial_{t} \int_{\mathbb{R}} \psi\left(\frac{x-v t}{R}\right) \mathbb{\mathbb { T }}_{01}(t, x) d x=-\frac{1}{R} \int_{\mathbb{R}} \chi\left(\frac{x-v t}{R}\right)\left(\mathbb{T}_{11}(t, x)+v \mathbb{\mathbb { T }}_{01}(t, x)\right) d x ;
$$

integrating this against $\chi(t / T)$ using (7) we conclude that

$$
\int_{\mathbb{R}} \int_{\mathbb{R}} \chi\left(\frac{t}{T}\right) \chi\left(\frac{x-v t}{R}\right)\left(\mathbb{T}_{11}(t, x)+v \mathbb{\mathbb { T }}_{01}(t, x)\right) d x d t=O(R) .
$$

A similar argument using (3) instead of (4) yields

$$
\int_{\mathbb{R}} \int_{\mathbb{R}} \chi\left(\frac{t}{T}\right) \chi\left(\frac{x-v t}{R}\right)\left(\mathbb{T}_{01}(t, x)+v \mathbb{\mathbb { T }}_{00}(t, x)\right) d x d t=O(R) .
$$

On the other hand, if we define the nonlinear null form

$$
Q:=\left(-\partial_{t t}+\partial_{x x}\right) u^{2}=-2 u_{t}^{2}+2 u_{x}^{2}+2|u|^{p+1}
$$

then from integration by parts and (8) we have

$$
\begin{aligned}
\left|\int_{\mathbb{R}} \int_{\mathbb{R}} \chi\left(\frac{t}{T}\right) \chi\left(\frac{x-v t}{R}\right) Q(t, x) d x d t\right| & =\left|\int_{\mathbb{R}} \int_{\mathbb{R}} u^{2}(t, x)\left(-\partial_{t t}+\partial_{x x}\right)\left(\chi\left(\frac{t}{T}\right) \chi\left(\frac{x-v t}{R}\right)\right) d x d t\right| \\
& \lesssim \int_{-2 T}^{2 T} \int_{v-2 R}^{v+2 R} \frac{1}{T^{2}}+\frac{1}{R^{2}} d x d t \\
& \lesssim \frac{R}{T}+\frac{T}{R} \lesssim \frac{T}{R} .
\end{aligned}
$$

Let us compare $|u|^{p+1}$ against the quantities

$$
\begin{aligned}
& \mathbb{T}_{11}+v \mathbb{\mathbb { T }}_{01}=\frac{1}{2} u_{t}^{2}+v u_{t} u_{x}+\frac{1}{2} u_{x}^{2}-\frac{1}{p+1}|u|^{p+1}, \\
& \mathbb{T}_{01}+v \mathbb{\varpi}_{00}=\frac{1}{2} v u_{t}^{2}+u_{t} u_{x}+\frac{1}{2} v u_{x}^{2}+\frac{v}{p+1}|u|^{p+1}, \\
& Q=-2 u_{t}^{2}+2 u_{x}^{2}+2|u|^{p+1} .
\end{aligned}
$$


We divide into three cases.

Case 1 (spacelike): $v \geq 1$. In this case, we can verify the pointwise bound

$$
\frac{1}{p+1}|u|^{p+1} \leq \mathbb{T}_{01}+v \mathbb{\mathbb { T }}_{00}
$$

and so (11) follows immediately from (13) (note that $R=O\left(R^{1 / 2} T^{1 / 2}\right)$ ).

Case 2 (lightlike): $1-\frac{R^{1 / 2}}{2 T^{1 / 2}}<v<1$. In this case we have the bound

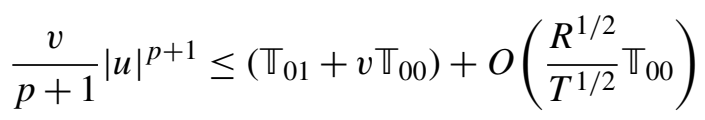

and so from (13) and (7) we have

$$
\frac{v}{p+1} \int_{\mathbb{R}} \int_{\mathbb{R}} \chi\left(\frac{t}{T}\right) \chi\left(\frac{x-v t}{R}\right)|u(t, x)|^{p+1} d t d x \lesssim R+R^{1 / 2} T^{1 / 2}
$$

and (11) follows.

Case 3 (timelike): $0 \leq v \leq 1-\frac{R^{1 / 2}}{2 T^{1 / 2}}$. Here we use the identity

$$
\left(\mathbb{\mathbb { V }}_{11}+v \mathbb{\mathbb { T }}_{01}\right)-v\left(\mathbb{\mathbb { T }}_{01}+v \mathbb{\mathbb { T }}_{00}\right)+\frac{1-v^{2}}{4} Q=\left(1-v^{2}\right) u_{x}^{2}+\frac{(p-1)\left(1-v^{2}\right)}{2(p+1)}|u|^{p+1} .
$$

Taking the indicated linear combination of (12), (13), (14) and discarding $\left(1-v^{2}\right) u_{x}^{2}$, which is nonnegative, we conclude that

$$
\frac{(p-1)\left(1-v^{2}\right)}{2(p+1)} \int_{\mathbb{R}} \int_{\mathbb{R}} \chi\left(\frac{t}{T}\right) \chi\left(\frac{x-v t}{R}\right)|u(t, x)|^{p+1} d t d x \lesssim R+\frac{1-v^{2}}{4} \frac{T}{R}
$$

and thus (noting that $1-v^{2}=(1-v)(1+v)$ is comparable to $1-v$ )

$$
\int_{\mathbb{R}} \int_{\mathbb{R}} \chi\left(\frac{t}{T}\right) \chi\left(\frac{x-v t}{R}\right)|u(t, x)|^{p+1} d t d x \lesssim \frac{R}{1-v}+\frac{T}{R} .
$$

Since $1-v \gtrsim R^{1 / 2} / T^{1 / 2}$ by hypothesis, the claim follows.

\section{Proof of Theorem 1.1}

We are now ready to prove Theorem 1.1. Suppose that this claim failed for some $E, p$. Carefully negating the quantifiers, we may thus find a sequence of times $T_{n} \rightarrow \infty$ and $t_{n} \in \mathbb{R}$, a $\delta>0$ independent of $n$, and a family of solutions $u_{n}$ which uniformly obey the energy bound $E\left[u_{n}\right] \leq E$ such that

$$
\frac{1}{2 T_{n}} \int_{t_{n}-T_{n}}^{t_{n}+T_{n}}\left\|u_{n}(t)\right\|_{L_{x}^{\infty}(\mathbb{R})} d t \geq \delta
$$

By translating each $u_{n}$ by $t_{n}$, we may normalise $t_{n}=0$. 
Let $n$ be large. We will now allow our implied constants in the $\lesssim$ notation to depend on $\delta$, thus

$$
\int_{-T_{n}}^{T_{n}}\left\|u_{n}(t)\right\|_{L^{\infty}(\mathbb{R})} d t \gtrsim T_{n}
$$

From this bound and (8), we now conclude that the set

$$
\left\{t \in\left[-T_{n}, T_{n}\right]:\left\|u_{n}(t)\right\|_{L^{\infty}(\mathbb{R})} \gtrsim 1\right\}
$$

has Lebesgue measure $\gtrsim T_{n}$ (for suitable choices of implied constants). In particular, we can find a finite set $\Delta_{n} \subset\left[-T_{n}, T_{n}\right]$ of times which are 1-separated and of cardinality

$$
\# \Delta_{n} \gtrsim T_{n}
$$

such that

$$
\left\|u_{n}(t)\right\|_{L^{\infty}(\mathbb{R})} \gtrsim 1
$$

for all $t \in \Delta_{n}$.

For each $t \in \Delta_{n}$, let $x_{n}(t) \in \mathbb{R}$ be a point such that $\left|u_{n}\left(t, x_{n}(t)\right)\right| \geq \frac{1}{2}\left\|u_{n}(t)\right\|_{L^{\infty}(\mathbb{R})}$. From (15), one has

$$
\left|u_{n}\left(t, x_{n}(t)\right)\right| \gtrsim 1
$$

for all $t \in \Delta_{n}$.

Let us say that two times $t, t^{\prime} \in \Delta_{n}$ are spacelike if we have

$$
\left|x_{n}\left(t^{\prime}\right)-x_{n}(t)\right| \geq\left|t-t^{\prime}\right|+1 .
$$

There is a limit as to how many spacelike pairs of times can exist:

Lemma 3.1 (finite speed of propagation). Let $n$ be sufficiently large, and let $t_{1}, \ldots, t_{m} \in \Delta_{n}$ be times which are pairwise spacelike. Then we have $m=O(1)$.

Proof. Without loss of generality we may assume that $t_{1}<\ldots<t_{m}$. Consider the spacetime region

$$
\Omega:=\mathbb{R} \times \mathbb{R} \backslash \bigcup_{1 \leq j \leq m}\left\{(t, x): t \geq t_{j} \text { and }\left|x-x_{n}\left(t_{j}\right)\right| \leq t-t_{j}+\frac{1}{2}\right\} .
$$

Standard energy estimates reveal that

$$
\int_{\left(t_{j}, x\right) \in \Omega} \mathbb{T}_{00}\left(t_{j}, x\right) d x+\int_{\left|x-x_{n}\left(t_{j}\right)\right| \leq \frac{1}{2}} \mathbb{T}_{00}\left(t_{j}, x\right) d x \leq \int_{\left(t_{j-1}, x\right) \in \Omega} \mathbb{T}_{00}\left(t_{j-1}, x\right) d x
$$

for all $1<j \leq m$, where $\mathbb{T}_{00}=\mathbb{T}_{00, n}$ is the energy density of $u_{n}$. Iterating this and then using (7), we conclude that

$$
\sum_{1<j \leq m} \int_{\left|x-x_{n}\left(t_{j}\right)\right| \leq \frac{1}{2}} \mathbb{T}_{00}\left(t_{j}, x\right) d x \lesssim 1
$$

and in particular that

$$
\sum_{1<j \leq m} \int_{\left|x-x_{n}\left(t_{j}\right)\right| \leq \frac{1}{2}}\left|u_{n}\left(t_{j}, x\right)\right|^{p+1} d x \lesssim 1
$$


But from (16), (9) we see that

$$
\int_{\left|x-x_{n}\left(t_{j}\right)\right| \leq \frac{1}{2}}\left|u_{n}\left(t_{j}, x\right)\right|^{p+1} d x \gtrsim 1 .
$$

for each $j$, and the claim follows.

We now use this lemma and some combinatorial arguments to extract a Lipschitz worldline.

Corollary 3.2 (existence of Lipschitz worldline). Let $\varepsilon_{0}:(0,1] \rightarrow(0,1]$ be an arbitrary function. Then there exists a constant $0<c_{0}=c_{0}\left(\varepsilon_{0}\right) \leq 1$ with the following property: for all sufficiently large $n$, there exists $c_{0}<c<1$ (depending on $n$ ) and a subset $\Delta_{n}^{\prime}$ of $\Delta_{n}$ with

$$
\# \Delta_{n}^{\prime} \geq c T_{n}
$$

such that we have the Lipschitz property

$$
\left|x_{n}\left(t^{\prime}\right)-x_{n}(t)\right| \leq\left|t-t^{\prime}\right|+\varepsilon_{0}(c) T_{n}
$$

for all $t, t^{\prime} \in \Delta_{n}^{\prime}$.

Proof. Fix $\varepsilon$, and let $n$ be sufficiently large. Define the particle number of a set $\Delta$ to be the largest integer $m$ for which one can find pairwise spacelike times $t_{1}, \ldots, t_{m}$ in $\Delta$. By the previous lemma, we see that $\Delta_{n}$ has particle number $O(1)$. The key lemma is the following:

Lemma 3.3 (dichotomy). Let $\Delta^{\prime} \subset \Delta_{n}, m=O(1)$ and $c>0$ be such that

$$
\# \Delta^{\prime} \geq 2 c T_{n}
$$

and $\Delta^{\prime}$ has particle number at most $m$. Suppose $n$ is sufficiently large depending on $c$. Then at least one of the following is true:

(i) There exists a subset $\Delta^{\prime \prime} \subset \Delta^{\prime}$ of cardinality at least $c T_{n}$ such that (17) holds for all $t, t^{\prime} \in \Delta^{\prime \prime}$.

(ii) There exists a subset $\Delta^{\prime \prime \prime} \subset \Delta^{\prime}$ of cardinality at least $c \varepsilon_{0}(c) T_{n} / 16$ with particle number at most $m-1$.

Iterating this lemma at most $O(1)$ times we obtain the claim.

It remains to prove the lemma. We subdivide the interval $\left[-T_{n}, T_{n}\right]$ into intervals $I$ of length between $\varepsilon_{0}(c) T_{n} / 4$ and $\varepsilon_{0}(c) T_{n} / 8$. Call an interval sparse if $\#\left(\Delta^{\prime} \cap I\right) \leq c \varepsilon_{0}(c) T_{n} / 8$, and dense otherwise. Observe that at most $c T_{n}$ elements of $\Delta^{\prime}$ lie in sparse intervals. Thus if we let $\Delta^{\prime \prime}$ denote the intersection of $\Delta^{\prime}$ with the union of all the dense intervals, then $\# \Delta^{\prime \prime} \geq c T_{n}$.

If $\Delta^{\prime \prime}$ obeys (17) then we are done. Otherwise, we can find $t_{1}, t_{2} \in \Delta^{\prime \prime}$ such that

$$
\left|x_{n}\left(t_{1}\right)-x_{n}\left(t_{2}\right)\right|>\left|t_{1}-t_{2}\right|+\varepsilon_{0}(c) T_{n} .
$$

The time $t_{1}$ must lie in some dense interval $I$. We split $\Delta^{\prime \prime} \cap I=\Delta_{1}^{\prime \prime \prime} \cup \Delta_{2}^{\prime \prime \prime}$, where $\Delta_{1}^{\prime \prime \prime}$ consists of all $t \in \Delta^{\prime \prime} \cap I$ with $\left|x_{n}(t)-x_{n}\left(t_{1}\right)\right| \leq \varepsilon_{0}(c) T_{n} / 2$, and $\Delta_{2}^{\prime \prime \prime}$ consists of the remainder of $\Delta^{\prime \prime} \cap I$. Observe from the triangle inequality (if $n$ is sufficiently large depending on $c$ ) that all times in $\Delta_{1}^{\prime \prime \prime}$ are spacelike with respect to $t_{2}$, and similarly all times in $\Delta_{2}^{\prime \prime \prime}$ are spacelike with respect to $t_{1}$. Thus each of $\Delta_{1}^{\prime \prime \prime}$ and $\Delta_{2}^{\prime \prime \prime}$ can 
have particle number at most $m-1$. On the other hand, by the pigeonhole principle, one of $\Delta_{1}^{\prime \prime \prime}$ and $\Delta_{2}^{\prime \prime \prime}$ must have cardinality at least $\frac{1}{2} \#\left(\Delta^{\prime \prime} \cap I\right)$, which is at least $c \varepsilon_{0}(c) T_{n} / 16$ since $I$ is dense. The lemma, and hence the corollary, follows.

Let $\varepsilon_{0}:(0,1] \rightarrow(0,1]$ to be a function to be chosen later (one should think of $\varepsilon_{0}(c)$ as going to zero very rapidly as $c \rightarrow 0$ ). For any sufficiently large $n$, let $c_{0}, c$ and $\Delta_{n}^{\prime}$ be as in Corollary 3.2.

Define the function $x_{n}^{\prime}:\left[-T_{n}, T_{n}\right] \rightarrow \mathbb{R}$ by

$$
x_{n}^{\prime}(t):=\inf _{t^{\prime} \in \Delta_{n}^{\prime}}\left(x_{n}\left(t^{\prime}\right)-\left|t-t^{\prime}\right|\right) .
$$

One easily verifies that $x_{n}^{\prime}$ is Lipschitz with constant at most 1 . From (17) we also see that

$$
\left|x_{n}(t)-x_{n}^{\prime}(t)\right| \leq \varepsilon_{0}(c) T_{n}
$$

for all $t \in \Delta_{n}^{\prime}$.

We now apply a quantitative version of the Rademacher (or Lebesgue) differentiation theorem to ensure that $x_{n}^{\prime}(t)$ is approximately differentiable on a large interval.

Proposition 3.4 (quantitative Rademacher differentiation theorem). Let $\varepsilon_{1}:(0,1] \rightarrow(0,1]$ be a function, and let $\delta>0$. Then there exists $r_{1}=r_{1}\left(\varepsilon_{1}, \delta\right)>0$ with the following property: given any Lipschitz function $f:[-1,1] \rightarrow \mathbb{R}$ with Lipschitz constant at most 1 , there exists $r_{1} \leq r \leq 1$ such that the set

$\left\{x \in[-1,1]\right.$ : there exists $L \in \mathbb{R}$ such that $\left|\frac{f(y)-f(x)}{y-x}-L\right| \leq \delta$

whenever $y \in[-1,1]$ is such that $\left.\varepsilon_{1}(r) \leq|y-x| \leq r\right\}$

(which, intuitively, is the set where $f$ is approximately differentiable) has Lebesgue measure at least $2-\delta$.

Proof. We give an indirect "compactness and contradiction" proof. Suppose for contradiction that the claim failed. Negating the quantifiers carefully, this means that there exists a function $\varepsilon_{1}:(0,1] \rightarrow(0,1]$, a $\delta>0$, a sequence $r_{n} \rightarrow 0$, and a sequence $f_{n}:[0,1] \rightarrow \mathbb{R}$ of Lipschitz functions with constant at most 1 , such that the sets

$\left\{x \in[-1,1]:\right.$ there exists $L \in \mathbb{R}$ such that $\left|\frac{f_{n}(y)-f_{n}(x)}{y-x}-L\right| \leq \delta$

whenever $y \in[-1,1]$ is such that $\left.\varepsilon_{1}(r) \leq|y-x| \leq r\right\}$

have Lebesgue measure at most $2-\delta$ for all $n$ and all $r_{n} \leq r \leq 1$.

By translating each $f_{n}$ by a constant if necessary, we may assume that $f_{n}(0)=0$. The Lipschitz functions then form a bounded equicontinuous family on the compact domain $[-1,1]$, and so by the Arzelà-Ascoli theorem we may (after passing to a subsequence if necessary) assume that the $f_{n}$ converge 
uniformly to a limit $f$. We conclude that the set

$$
\begin{aligned}
& \left\{x \in[-1,1]: \text { there exists } L \in \mathbb{R} \text { such that }\left|\frac{f(y)-f(x)}{y-x}-L\right| \leq \delta / 2\right. \\
& \text { whenever } \left.y \in[-1,1] \text { is such that } \varepsilon_{1}(r) \leq|y-x| \leq r\right\}
\end{aligned}
$$

has Lebesgue measure at most $2-\delta$ for all $0<r \leq 1$. On the other hand, $f$ is clearly Lipschitz with constant at most 1 , and so by the Lipschitz differentiation theorem, $f$ is differentiable almost everywhere. In particular, the set

$$
\bigcup_{m=1}^{\infty}\left\{x \in[-1,1]: \text { there exists } L \in \mathbb{R} \text { such that }\left|\frac{f(y)-f(x)}{y-x}-L\right| \leq \delta / 2\right.
$$

whenever $y \in[-1,1]$ is such that $\left.0 \leq|y-x| \leq 2^{-m}\right\}$

has full measure in $[-1,1]$. By the monotone convergence theorem, this implies that one of the sets in this union has measure greater than $2-\delta$. But this contradicts the previous claim.

Remark 3.5. It is also possible to give a more direct "martingale" 2 or "multiscale analysis" proof of this proposition, which we sketch as follows. For each $n \geq 1$, let $f_{n}$ be the piecewise linear continuous function which agrees with $f$ on multiples of $2^{-n}$, and is linear between such intervals. One easily verifies that the functions $f_{n+1}-f_{n}$ are pairwise orthogonal in the Hilbert space $\dot{H}^{1}([-1,1])$, and thus by Bessel's inequality we have

$$
\sum_{n=1}^{\infty}\left\|f_{n+1}-f_{n}\right\|_{\dot{H}^{1}([-1,1])}^{2} \leq 2
$$

Now let $F: \mathbb{N} \rightarrow \mathbb{N}$ be a function to be chosen later, and let $\sigma>0$ be a small quantity to be chosen later. From the pigeonhole principle, one can find $1 \leq n_{0} \leq C(F, \sigma)$ such that

$$
\sum_{n=n_{0}}^{F\left(n_{0}\right)}\left\|f_{n+1}-f_{n}\right\|_{\dot{H}^{1}([-1,1])}^{2} \leq \sigma .
$$

If one then sets $r:=\sigma 2^{-n_{0}}$, one can verify all the required claims if $\sigma$ is chosen sufficiently small depending on $\delta$, and $F$ is sufficiently rapidly growing depending on $\delta, \sigma$, and $\varepsilon_{0}$; the quantity $L$ can basically be taken to be $f_{n}^{\prime}(x)$. We omit the details, but see [Tao 2009] for some similar arguments in this spirit.

Let $\delta>0$ be a small quantity (depending on $c$ ) to be chosen later, and let $\varepsilon_{1}:(0,1] \rightarrow(0,1]$ be the function $\varepsilon_{1}(r):=\delta r$. We let $n$ be sufficiently large, and apply the above proposition to the Lipschitz function $f=f_{n}:[-1,1] \rightarrow \mathbb{R}$ defined by $f(y):=1 / T_{n} x_{n}^{\prime}\left(T_{n} y\right)$. We conclude that there exists $r_{1}=r_{1}(\delta)$

\footnotetext{
${ }^{2}$ Indeed, the arguments here are closely related to some classical martingale inequalities of Doob [1953] and Lépingle [1976].
} 
and $r_{1}<r<1$ (depending on $\delta$ and $n$ ) such that the set

$$
\begin{array}{r}
\left\{x \in\left[-T_{n}, T_{n}\right]: \text { there exists } L \in \mathbb{R} \text { such that }\left|\frac{x_{n}^{\prime}\left(t^{\prime}\right)-x_{n}^{\prime}(t)}{t^{\prime}-t}-L\right| \leq \delta\right. \\
\text { whenever } \left.y \in\left[-T_{n}, T_{n}\right] \text { is such that } \delta r T_{n} \leq\left|t-t^{\prime}\right| \leq r T_{n}\right\}
\end{array}
$$

has measure at least $(2-\delta) T_{n}$.

On the other hand, the set $\Delta_{n}^{\prime}$ has cardinality at least $c T_{n}$. As in the proof of Lemma 3.3, we partition $\left[-T_{n}, T_{n}\right]$ into intervals $I$ of length between $r T_{n} / 4$ and $r T_{n} / 8$, and let $\Delta_{n}^{\prime \prime}$ be the portion of $\Delta_{n}^{\prime}$ which are contained inside those intervals $I$ which are dense in the sense that they contain at least $c r T_{n} / 16$ elements of $\Delta_{n}^{\prime}$. It is easy to see that $\Delta_{n}^{\prime \prime}$ has cardinality at least $c T_{n} / 2$. Also, $\Delta_{n}^{\prime \prime}$ is 1 -separated.

Thus, if we let $\delta=\delta(c)$ be sufficiently small compared to $c$, we can find $t_{*} \in\left[-T_{n}, T_{n}\right]$ within a distance 1 of $\Delta_{n}^{\prime \prime}$ and $v \in \mathbb{R}$ such that

$$
\left|\frac{x_{n}^{\prime}\left(t^{\prime}\right)-x_{n}^{\prime}\left(t_{*}\right)}{t^{\prime}-t_{*}}-v\right| \leq \delta \quad \text { whenever } t^{\prime} \in\left[-T_{n}, T_{n}\right] \text { is such that } \delta r T_{n} \leq\left|t_{*}-t^{\prime}\right| \leq r T_{n} .
$$

Let $t_{0}$ be an element of $\Delta_{n}^{\prime \prime}$ within 1 of $t_{*}$. Applying (18), the triangle inequality, and the Lipschitz nature of $x_{n}^{\prime}$, we conclude that

$$
x_{n}\left(t_{1}\right)=x_{n}\left(t_{0}\right)+v\left(t_{1}-t_{0}\right)+O\left(\delta\left|t_{1}-t_{0}\right|\right)+O\left(\varepsilon_{0}(c) T_{n}\right)+O(1)
$$

whenever $t_{1} \in \Delta_{n}^{\prime \prime}$ is such that $\delta T_{n}+1 \leq\left|t_{1}-t_{0}\right| \leq r T_{n}-1$. Applying the Lipschitz property again, we conclude that

$$
x_{n}\left(t_{1}\right)=x_{n}\left(t_{0}\right)+v\left(t_{1}-t_{0}\right)+O\left(\delta r T_{n}\right)+O\left(\varepsilon_{0}(c) T_{n}\right)+O(1)
$$

for all $t_{1} \in \Delta_{n}^{\prime \prime}$ with $\left|t_{1}-t_{0}\right| \leq r T_{n}-1$. If we set $\varepsilon_{0}(c):=\delta(c) r_{1}(\delta(c))$, and assume $n$ is sufficiently large depending on all other parameters, we thus have

$$
x_{n}\left(t_{1}\right)=x_{n}\left(t_{0}\right)+v\left(t_{1}-t_{0}\right)+O\left(\delta r T_{n}\right)
$$

whenever $t_{1} \in \Delta_{n}^{\prime \prime}$ and $\left|t_{1}-t_{0}\right| \leq r T_{n} / 4$. One should view this as an assertion that $x_{n}$ is approximately differentiable near $t_{0}$.

By definition of $\Delta_{n}^{\prime \prime}$, we know that $t_{0}$ is contained in an interval $I$ of length at most $r T_{n} / 4$ which contains $\gtrsim c r T_{n}$ elements of $\Delta_{n}$. We thus see that the parallelogram

$$
P:=\left\{(t, x): t \in I,\left|x-x_{n}\left(t_{0}\right)-v\left(t-t_{0}\right)\right| \leq R / 2\right\}
$$

contains at least $\gtrsim c r T_{n}$ points of the form $\left(t, x_{n}(t)\right)$ with $t \in \Delta_{n}$, where $R$ is a quantity of size $\sim \delta r T_{n}$. On the other hand, by definition of $\Delta_{n}$, we have $\left|u_{n}(t, x(t))\right| \gtrsim 1$ for all $t \in \Delta_{n}$. Applying (9), we conclude that

$$
\int_{P}\left|u_{n}(t, x)\right|^{p+1} d t d x \gtrsim \operatorname{cr} T_{n}
$$


On the other hand, from Proposition 2.2 we have

$$
\int_{P}\left|u_{n}(t, x)\right|^{p+1} d t d x \lesssim R^{1 / 2}\left(r T_{n}\right)^{1 / 2}+\frac{r T_{n}}{R} \lesssim \delta^{1 / 2} r T_{n}+\delta^{-1} .
$$

If we set $\delta$ to be sufficiently small depending on $c$, and let $n$ be sufficiently large depending on all other parameters, we obtain a contradiction as desired. This completes the proof of Theorem 1.1.

\section{Acknowledgement}

We thank Jason Murphy, Qing Tian Zhang and the anonymous referee for corrections.

\section{References}

[Doob 1953] J. L. Doob, Stochastic processes, Wiley, New York, 1953. MR 15,445b Zbl 0053.26802

[Lépingle 1976] D. Lépingle, "La variation d'ordre $p$ des semi-martingales", Z. Wahrscheinlichkeitstheorie und Verw. Gebiete 36:4 (1976), 295-316. MR 54 \#8849 Zbl 0325.60047

[Lindblad and Soffer 2005] H. Lindblad and A. Soffer, "A remark on asymptotic completeness for the critical nonlinear KleinGordon equation”, Lett. Math. Phys. 73:3 (2005), 249-258. MR 2006i:35249 Zbl 1106.35072

[Reed 1978] M. C. Reed, "Propagation of singularities for non-linear wave equations in one dimension", Comm. Partial Differential Equations 3:2 (1978), 153-199. MR 80d:35092 Zbl 0377.35047

[Tao 2008] T. Tao, Structure and randomness: pages from year one of a mathematical blog, American Mathematical Society, Providence, RI, 2008. MR 2010h:00002 Zbl 05380664

[Tao 2009] T. Tao, "A quantitative version of the Besicovitch projection theorem via multiscale analysis", Proc. Lond. Math. Soc. (3) 98:3 (2009), 559-584. MR 2010f:28009 Zbl 1173.28001

Received 3 Nov 2010. Revised 12 Jan 2011. Accepted 7 Feb 2011.

HANS LINDBLAD: lindblad@math.ucsd.edu

Department of Mathematics, University of California, San Diego, La Jolla, CA 92093-0112, United States

TERENCE TAO: tao@math.ucla.edu

Department of Mathematics, University of California, Los Angeles, 405 Hilgard Avenue, Los Angeles, CA 90095-1555, United States 


\title{
Analysis \& PDE
}

\author{
msp.berkeley.edu/apde
}

EDITORS

EDITOR-IN-CHIEF

Maciej Zworski

University of California

Berkeley, USA

BOARD OF EDITORS

\begin{tabular}{|c|c|c|c|}
\hline Michael Aizenman & $\begin{array}{l}\text { Princeton University, USA } \\
\text { aizenman@math.princeton.edu }\end{array}$ & Nicolas Burq & $\begin{array}{l}\text { Université Paris-Sud 11, France } \\
\text { nicolas.burq@math.u-psud.fr }\end{array}$ \\
\hline Luis A. Caffarelli & $\begin{array}{l}\text { University of Texas, USA } \\
\text { caffarel@math.utexas.edu }\end{array}$ & un-Yung Alice Chang & $\begin{array}{l}\text { Princeton University, USA } \\
\text { chang@math.princeton.edu }\end{array}$ \\
\hline Michael Christ & $\begin{array}{l}\text { University of California, Berkeley, USA } \\
\text { mchrist@math.berkeley.edu }\end{array}$ & Charles Fefferman & $\begin{array}{l}\text { Princeton University, USA } \\
\text { cf@math.princeton.edu }\end{array}$ \\
\hline Ursula Hamenstaedt & $\begin{array}{l}\text { Universität Bonn, Germany } \\
\text { ursula@math.uni-bonn.de }\end{array}$ & Nigel Higson & $\begin{array}{l}\text { Pennsylvania State Univesity, USA } \\
\text { higson@ math.psu.edu }\end{array}$ \\
\hline Vaughan Jones & $\begin{array}{l}\text { University of California, Berkeley, USA } \\
\text { vfr@math.berkeley.edu }\end{array}$ & Herbert Koch & $\begin{array}{l}\text { Universität Bonn, Germany } \\
\text { koch@math.uni-bonn.de }\end{array}$ \\
\hline Izabella Laba & $\begin{array}{l}\text { University of British Columbia, Canada } \\
\text { ilaba@math.ubc.ca }\end{array}$ & Gilles Lebeau & $\begin{array}{l}\text { Université de Nice Sophia Antipolis, France } \\
\text { lebeau@unice.fr }\end{array}$ \\
\hline László Lempert & $\begin{array}{l}\text { Purdue University, USA } \\
\text { lempert@math.purdue.edu }\end{array}$ & Richard B. Melrose & $\begin{array}{l}\text { Massachussets Institute of Technology, USA } \\
\text { rbm@math.mit.edu }\end{array}$ \\
\hline Frank Merle & $\begin{array}{l}\text { Université de Cergy-Pontoise, France } \\
\text { Frank.Merle@u-cergy.fr }\end{array}$ & William Minicozzi II & $\begin{array}{l}\text { Johns Hopkins University, USA } \\
\text { minicozz@ math.jhu.edu }\end{array}$ \\
\hline Werner Müller & $\begin{array}{l}\text { Universität Bonn, Germany } \\
\text { mueller@math.uni-bonn.de }\end{array}$ & Yuval Peres & $\begin{array}{l}\text { University of California, Berkeley, USA } \\
\text { peres@stat.berkeley.edu }\end{array}$ \\
\hline Gilles Pisier & $\begin{array}{l}\text { Texas A\&M University, and Paris } 6 \\
\text { pisier@math.tamu.edu }\end{array}$ & Tristan Rivière & $\begin{array}{l}\text { ETH, Switzerland } \\
\text { riviere@math.ethz.ch }\end{array}$ \\
\hline Igor Rodnianski & $\begin{array}{l}\text { Princeton University, USA } \\
\text { irod@math.princeton.edu }\end{array}$ & Wilhelm Schlag & $\begin{array}{l}\text { University of Chicago, USA } \\
\text { schlag@math.uchicago.edu }\end{array}$ \\
\hline Sylvia Serfaty & $\begin{array}{l}\text { New York University, USA } \\
\text { serfaty@ cims.nyu.edu }\end{array}$ & Yum-Tong Siu & $\begin{array}{l}\text { Harvard University, USA } \\
\text { siu@math.harvard.edu }\end{array}$ \\
\hline Terence Tao & $\begin{array}{l}\text { University of California, Los Angeles, USA } \\
\text { tao@math.ucla.edu }\end{array}$ & A Michael E. Taylor & $\begin{array}{l}\text { Univ. of North Carolina, Chapel Hill, USA } \\
\text { met@math.unc.edu }\end{array}$ \\
\hline Gunther Uhlmann & $\begin{array}{l}\text { University of Washington, USA } \\
\text { gunther@math.washington.edu }\end{array}$ & András Vasy & $\begin{array}{l}\text { Stanford University, USA } \\
\text { andras@math.stanford.edu }\end{array}$ \\
\hline Virgil Voiculescu & $\begin{array}{l}\text { University of California, Berkeley, USA } \\
\text { dvv@math.berkeley.edu }\end{array}$ & Steven Zelditch & $\begin{array}{l}\text { Northwestern University, USA } \\
\text { zelditch@math.northwestern.edu }\end{array}$ \\
\hline
\end{tabular}

\section{PRODUCTION}

contact@msp.org

Silvio Levy, Scientific Editor

Sheila Newbery, Senior Production Editor

See inside back cover or msp.berkeley.edu/apde for submission instructions.

The subscription price for 2012 is US \$140/year for the electronic version, and \$240/year for print and electronic. Subscriptions, requests for back issues from the last three years and changes of subscribers address should be sent to Mathematical Sciences Publishers, Department of Mathematics, University of California, Berkeley, CA 94720-3840, USA.

Analysis \& PDE, at Mathematical Sciences Publishers, Department of Mathematics, University of California, Berkeley, CA 94720-3840 is published continuously online. Periodical rate postage paid at Berkeley, CA 94704, and additional mailing offices.

APDE peer review and production are managed by EditFLOW ${ }^{\mathrm{TM}}$ from Mathematical Sciences Publishers.

PUBLISHED BY

mathematical sciences publishers

http://msp.org/

A NON-PROFIT CORPORATION

Typeset in IATEX

Copyright $(2012$ by Mathematical Sciences Publishers 


\section{ANALYSIS \& PDE}

\section{Volume $5 \quad$ No. $2 \quad 2012$}

The geodesic X-ray transform with fold caustics

Plamen STEFANOV and GUNTHER UHLMANN

Existence of extremals for a Fourier restriction inequality

Michael Christ and Shuanglin ShaO

Dispersion and controllability for the Schrödinger equation on negatively curved manifolds

NALINI ANANTHARAMAN and GABRIEL RIVIÈRE

A bilinear oscillatory integral estimate and bilinear refinements to Strichartz estimates on closed manifolds

\section{ZAHER HANI}

The Cauchy problem for the Benjamin-Ono equation in $L^{2}$ revisited

LUC MOLINET and DidIER PILOD

On triangles determined by subsets of the Euclidean plane, the associated bilinear operators 